\title{
Knowledge Based Product and Process Engineering Enabling Design and Manufacture Integration
}

\author{
Matthieu Bricogne, Farouk Belkadi, Magali Bosch-Mauchand, and Benoît Eynard \\ Université de Technologie de Compiègne, \\ Mechanical Systems Engineering Department, \\ CNRS UMR6253 Roberval \\ BP 60319, rue du Docteur Schweitzer, \\ 60203 Compiègne Cedex, France \\ \{matthieu.bricogne, farouk.belkadi, magali.bosch, \\ benoit. eynard\} @utc.fr
}

\begin{abstract}
After presenting reminders about product and process knowledge, this paper describes a specific knowledge called in this paper "shared knowledge", which is built from a mapping between both these knowledge. Then, a specific approach based on "workers" is proposed to extract data from the different IT components, to create this shared knowledge and to capitalize for the future development projects. Finally, different industrial examples are presented to illustrate the shared knowledge capitalization interest.
\end{abstract}

Keywords: PLM, MPM, CAD/CAE, ERP, Knowledge Sharing.

\section{Introduction}

Nowadays artifacts have to meet high performance functions integrating various technologies and numerous components. To cope with this increasing complexity, the development of new methods and techniques has lead to an improvement of the results of engineering activities.

Design process takes an increasingly high importance in the whole product development and industrialization. First, the design process is one of the most complex phases while designers generate new knowledge. They integrate heterogeneous existing knowledge and they transform a set of requirements and constraints into a technical solution. Second, the different choices and decisions taken during the design phase strongly impact the other development phases, the production steps and more generally the whole product lifecycle.

Two main types of knowledge can currently be considered to get a successful result for the design of a product: product and process knowledge. However, another kind of knowledge is crucial to guarantee consistency between the product design's decisions and decisions related to the process. This transversal knowledge, called in this paper "shared knowledge", can be built from the mapping between product and process knowledge. 
The purpose of this paper is to describe a new approach taking advantage of PLM (Product Lifecycle Management) systems for extracting product and process knowledge from different IT systems. This knowledge is used to generate the shared knowledge.

\section{Knowledge Management}

The knowledge management is an interactive process that aims to transfer the knowledge throughout its different life states [1] [2]; Sainter distinguishes six steps for the knowledge management process from the knowledge identification to the knowledge reuse [3]. Each of them involves several actors coming from various fields.

Product development project is a source of new knowledge. For the achievement of his activities, the expert needs to reuse existing knowledge for the generation of new knowledge, which has to be integrated back in the management cycle. There is a large range of knowledge that designers use to match the ever-increasing complexity of design problems. Generally, design knowledge can be classified into two categories: product knowledge and process knowledge [4]. The next sections present the description of both kinds of knowledge and their properties.

\subsection{Product Knowledge}

The commonly accepted approach for structuring product knowledge has been through the construction of Product Models [5]. Several knowledge models reflecting the characteristics of the product are developed in the literature. One of the most usual modelling approaches is the "Function-Behaviour-Structure" (FBS) [6]. Various models are developed as an extension of the FBS method to cope with specific needs [7].

Therefore, regarding to the diversity of product knowledge, five knowledge categories might be distinguished in the building of a product model:

- Function: describes a formalization of the users' requirements according to their needs.

- Structure: describes the technical architecture of the product. The most used representations of this knowledge are the eBOM and $\mathrm{mBOM}$ (engineering / manufacturing Bill of Material).

- Behaviour: describes with different formalisms how the product fulfils different functions to cope with the user need regarding the constraints of its various lifecycle phases.

- Properties: complete the definition of the product structure by a set of characteristics such as: material, geometry, technology, etc.

- Life situations: include all constraints generated from the interaction of the product with its environment in each phase of its lifecycle.

In order to represent and share explicit product's knowledge, experts use different information system's components. IS tools are used to store, manipulate and manage data and information. For example, requirements engineering tools propose different tools to assist the designer in the description and the structuring of the product's functions. PDM systems are used to manage the eBOM structure, constituted of components, parts and sub parts. The corresponding product's structure for manufacturing view $(\mathrm{mBOM})$ is stored in the ERP server. 


\subsection{Process Knowledge}

During a product development project, the associated process knowledge is created during the whole lifecycle and different IT systems are involved [8] [9]. It permits to define the contribution of each activity and related resources to the various project goals and to make the connection between the different processes. This knowledge is useful for the IT systems requirements definition and to drive the information sharing mechanisms.

The process knowledge definition is based on activity models: activities allow creating the link between products, resources (facilities, humans...) and their characteristics (behaviour, task, properties...). They structure and define the behaviour of the processes. An activity aggregates several kinds of knowledge such as sequences, functions, rules, states... [10]

In order to formalize this knowledge, several tools such as ERP modules, MPM system [11] and Virtual Factory tools are involved. PLM, ERP and MPM [12] systems are used to manage it (store, manipulate, organize and capitalize). The diversity and the heterogeneous data models of software increase the complexity of the knowledge management process.

In the product development process, two types of activities can be set to support the process knowledge: design activities and, manufacturing and process planning activities. Information's nature and skills requirements are used to differentiate them. In this context, the aim is to make relevant decisions on product and / or on process.

During industrialization process, manufacturing process knowledge is used to create manufacturing data: the resource allocation, the part masters, the $\mathrm{mBOM}$, the $\mathrm{NC} /$ Robot programs, the operation sequences, the manufacturing process sheets, the shop floor documents... The manufacturing knowledge is contained in standard and specific processes, the set of resources at disposal (human resources, machines, tools and tooling), the organisation of the manufacturing unit (work centres), the manufacturing know-how. All the knowledge aims to provide coherency between products, resources and processes [13].

So process knowledge has specific properties, such as: the company's specificities; expert knowledge that is owned by individual beings and provided by different departments (Design, Methods, Quality, Manufacturing, Purchasing ...); the necessity of statistical analyse and update for certain data; the fact that specific activities are not yet formalized and then cannot be involved in data capitalization. Moreover, process knowledge defines the mapping between the product and the value chain.

\subsection{PLM Approach and Knowledge Building}

One of the major properties of knowledge is the capacity to generate a new kind of knowledge from existing one, by using data stored in engineering systems and mapping methods [2] [14].

In the case of explicit knowledge, the information system encapsulates knowledge's representation. An efficient way to extract knowledge is to use communication and information functionalities provided by the different IT tools. Because of their distributed and multi IS connections properties [15], PLM approach gives more advantages to support the knowledge extraction stage. 
Indeed, PLM is defined as a systematic concept for the integrated management of all product and processes related information through the entire lifecycle, from the initial idea to end-of-life [16]. In [17], PLM is considered as a strategic business approach that applies a consistent set of business solutions in support to the collaborative creation, management, dissemination, and use of product information across the extended enterprise. At the technical level, the IT solutions to support PLM approach result from the integration of heterogeneous systems such as ERP, PDM and MPM [18], etc.

\section{Approach for Shared Knowledge Capitalization}

One of the biggest challenges for the companies when they integrate new methodology, new system or new software is to reduce the cost of the solution's deployment project and to minimize the disruption on people's way of working. One of the advantages of the proposed approach is the fact it will not disrupt the global Information System (IS) of the company. Whatever the software used, the way they are interconnected, the data models they are based on, the approach is able to adapt to any configuration. Unlike traditional approach, the purpose is not to integrate some data coming from specific software to another based on a different data model, or to try to define a generic data model able to make different software working together. So the purpose of this approach is to propose a flexible infrastructure (Fig. 1) which should take advantage of the different information system components and which should extract the necessary data for building the shared knowledge.

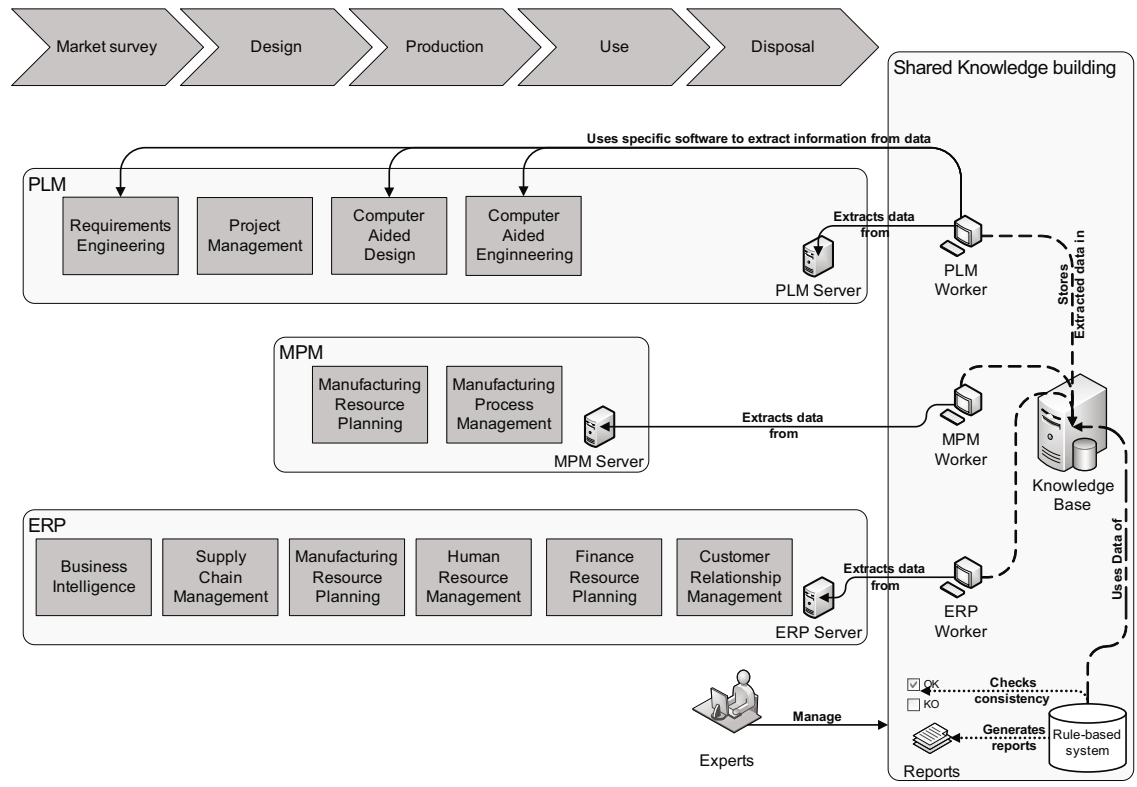

Fig. 1. Information system integration 


\subsection{Knowledge Extraction: The Workers}

For each involved information system's components, the goal is to extract the required data and to store it in the Knowledge Base (KB). This job is performed by a specific machine, called in this paper a worker. Each worker is dedicated to an IS component: it takes advantage of the extension's capabilities of every component in terms of chosen programming language, available Application Programming Interface (API), data model, etc... Sometimes, the data stored in the IS component need to be opened by specific software. In this case, this specific software can be installed in the worker in order to extract the desired information and to store it in the KB. For instance, in a PDM system, CAD data can be retrieved, opened and analyzed in order to store the proper information (e.g. the mass of the product) in the KB (Fig. 2).

In order to not disrupt the put in place components, the workers have to make their jobs during the periods of use less (for instance during the night if the system is not used in other countries).

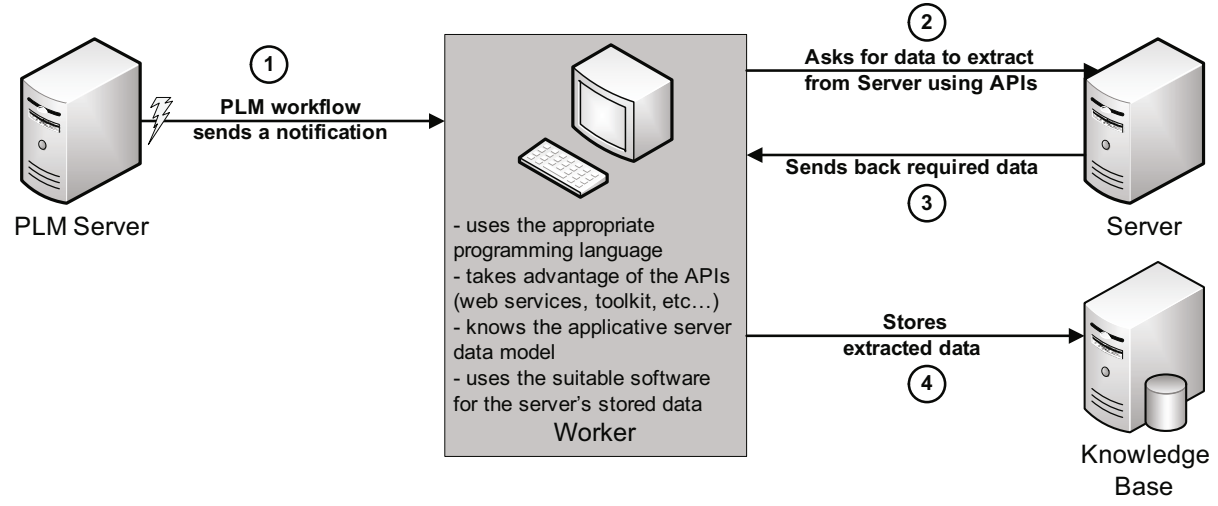

Fig. 2. Workers' processes for data extraction

\subsection{Knowledge Base Building and Update}

As explained above, the data retrieved from all the IS components thanks to the different workers are all stored in the KB. They can be some generic data, i.e. usable by the whole company for every project, or can be specific to a product, a product range, a step of a process, etc... The chosen structure for the heterogeneous information coming from the different systems is dependant of the company way of working and of the "shared knowledge deployment" goal.

This Knowledge Base can be a unique database or can be fragmented on several computer, for instance on the workers.

One of the most important challenges for this approach is to ensure that the data stored in the $\mathrm{KB}$ are up-to-date at the right moment. The update frequency is in-deed completely dependent of the data stored and of the product development / production stage. To pilot the updates of the different data, this paper suggests using the PLM workflow mechanism. The different workflows used send some "up-date required" 
notifications to the workers at the desired timing. Only necessary information is updated, avoiding surcharging the different system if it is not necessary.

\subsection{Shared Knowledge Building}

The way of using the extracted data constitutes the shared knowledge: it is a mapping between knowledge coming from different sectors of the company such as legal, research or manufacturing department. During every product's project progress, different meetings are organized in order to evaluate the different constraints of the stakeholders and to find solutions to the coming problems. A great part of the problems solved during these meetings could be avoided by formulating earlier the different actors' requirements. The most difficult work concerning this formulation is to give facts based on data stored in the different experts' software / applications. In order to extract the proper information, to analyze it and to use it, experts of the company's different departments must be brought together under the responsibility of shared knowledge capitalization project manager. They have to define the special data to match and to describe the links between these data. Finally, all these links should be formalized as constraints usable in the rule-based system.

The usage of a rule-based system is proposed to define the shared knowledge. It is not the unique manner to express this shared knowledge. Nevertheless it is probably one of the easiest way to put it in place because it consumes data, generates warnings, notifications and reports and has not for aim to generate other data.

This rule-based system is a central referential which can be manually sought or which can be launched by other applications. All the rules stored in the rule-based system have to be written in the same language but they should cover different fields to provide real benefits. The rules must correspond to the different constraints linked to a specific project, a specific standard, a specific plant, a specific range of products, etc...

\subsection{Case Study: Heavy and Civil Engineering Machines}

The main example of "shared knowledge capitalization" project deployment provided in this paper comes from a company working in the civil engineering and roadwork machines production. Its main job is to assemble the different machine parts comings from suppliers located all around the world. The purpose of the project was to manage the configuration of these machines depending on different objectives. The first goal was to respect the different standards imposed by the customer's legislation. The second intention was to propose to the customer the allowed marketing options. These options are mainly deduced from the legal and from the manufacturing (assembly line) constraints. The number of marketing options is huge, making it extremely difficult to manage.

The project has been started during the launch of a specific new machine. First of all, the legal department has to express the requirements linked to the specific standards they want to compliant with. These requirements have to be factual or numeric data in order to be easily usable by the rule-based system. Second, all the marketing options have to be mapped with the corresponding technical option(s). Third, the incompatible options have to be listed: the research department has to precise its 
constraints (mainly based on geometric models) and the manufacturing department has to clarify its restrictions according to its production's plans (e.g. 2 models of the same machine cannot be produced successively...).

Most of this knowledge is usable for other machines, other projects. It is a shared knowledge, a transversal knowledge. So it is important to store the application scope with the extracted data. For example, the scopes could be linked to: a prod-uct, a range of product, etc; a specific manufacturing plant, production line, tool used to assemble the product, etc; a specific standard concerning materials used or the requirements of the product, etc.

These data have also their own lifecycle and update timescale. This is why the PLM workflow could help to determine when the data has to be update depending on the project progress...

\section{Conclusion}

In this paper, the utility of the PLM approach has been underlined in order to support data extraction. The extracted data are used for the shared knowledge building. Shared knowledge is a new kind of knowledge, based on product and process knowledge. Its main purpose is to assess managerial and technical decisions.

The role of the experts still remains essential for this approach. For each involved information system's component, a key user has to be defined to determine which data is interesting to be extracted and to be used in the rule-based system. Then, they have to agree about the rules to define and the application field of these rules. The required competences for achieving such an implementation are multiple: software and network engineering to use the different provided APIs, different sections' expertise, project management to make all the experts working together, etc...

\section{References}

1. Liao, S.H.: Knowledge management technologies and applications - literature review from 1995 to 2002. Expert Systems with Applications 25, 155-164 (2003)

2. Studer, R., Benjamins, V.R., Fensel, D.: Knowledge Engineering: Principles and methods. Data and Knowledge Engineering 25, 161-197 (1998)

3. Sainter, P., Oldham, K., Larkin, A., Murton, A., Brimble, R.: Product Knowledge Management within Knowledge-Based engineering systems. In: ASME 2000 (2000)

4. Zha, X., Du, H.: Knowledge-intensive collaborative design modeling and support- Part I: Review, distributed models and framework. Comp. Ind. 57, 39-55 (2006)

5. Stokes, M.: Managing Engineering Knowledge: MOKA Methodology for Knowledge Based Engineering Applications. MOKA Consortium, London (2001)

6. Gero, J.S., Kannengiesser, U.: The situated function-behaviour-structure framework. Design Studies 25(4), 373-391 (2004)

7. Sudarsan, R., Fenves, S.F., Sriram, R.D., Wang, F.: Product information modeling framework for PLM. C.A.D. 37, 1399-1411 (2005)

8. Denkena, B., Shpitalni, M., Kowalski, P., Molcho, G., Zipori, Y.: Knowledge Management in Process Planning. CIRP Annals - Manufacturing Technology 56(1), 175-180 (2007) 
9. Ming, X.G., Yan, J.Q., Wang, X.H., Li, S.N., Lu, W.F., Peng, Q.J., Ma, Y.S.: Collaborative process planning and manufacturing in product lifecycle management. Comp. Ind. 59, 154-166 (2008)

10. Hugo, J., Vliegen, W., Herman, H.: Van Mal: The Structuring of Process Knowledge: Function, Task, Properties and State. Robotics \& Computer-Integrated Manufacturing 6(2), 101-107 (1989)

11. Huet, G., Fortin, C., Mcsorley, G., Toche, B.: Information Structures and Processes to Support Data Exchange between Product Development and Resource Planning Systems. In: IESM 2009, Inter. Conf. on Ind. Eng. and Syst. Mngt., Montreal, Canada, May 13-15 (2009)

12. Sly, D.: Manufacturing Process Management (MPM). Proplanner whitepaper (2004), http: / / www . proplanner. net/Product/Whitepapers /

13. Fortin, C., Huet, G.: Manufacturing Process Management: iterative synchronisation of engineering data with manufacturing realities. International Journal of Product Development 4(3/4), 280-295 (2007)

14. Gordon, J.L.: Creating knowledge maps by exploiting dependent relationships. Knowledge-Based Systems 13, 71-79 (2000)

15. Abramovici, M., Sieg, O.C.: Status and development trends of product lifecycle management systems. In: International Conference on Integrated Product and Process. Development - IPPD, Wroclaw, Poland, November 21-22 (2002)

16. Saaksvuori, A., Immonen, A.: PLM. Springer, Berlin (2004)

17. Jun, H., Kiritsis, D., Xirouchaki, P.: Research issues on closed-loop PLM. Computer in Industry 57 (2007)

18. Schuh, G., Rozenfeld, H., Assmus, D., Zancul, E.: Process oriented framework to support PLM implementation. Comp. Ind. 59, 210-218 (2008) 